\title{
The modal logic of continuous functions on cantor space
}

\author{
Philip Kremer
}

Received: 8 March 2004 / Published online: 27 September 2006

(C) Springer-Verlag 2006

\begin{abstract}
Let $\mathcal{L}$ be a propositional language with standard Boolean connectives plus two modalities: an S4-ish topological modality $\square$ and a temporal modality $\bigcirc$, understood as 'next'. We extend the topological semantic for S4 to a semantics for the language $\mathcal{L}$ by interpreting $\mathcal{L}$ in dynamic topological systems, i.e. ordered pairs $\langle X, f\rangle$, where $X$ is a topological space and $f$ is a continuous function on $X$. Artemov, Davoren and Nerode have axiomatized a logic S4C, and have shown that S4C is sound and complete for this semantics. Zhang and Mints have shown that $\mathrm{S} 4 \mathrm{C}$ is complete relative to a particular topological space, Cantor space. The current paper produces an alternate proof of the Zhang-Mints result.
\end{abstract}

\section{Introduction}

Let $\mathcal{L}^{\square}$ be a modal language with a set $P V=\left\{p_{1}, \ldots, p_{n}, \ldots\right\}$ of propositional variables; parentheses; connectives $\neg, \&$ and $\vee$; and a modal operator $\square$. The McKinsey-Tarski topological semantics [9,10], which predates Kripke's semantics [6,7], interprets $\mathcal{L}^{\square}$ in topological spaces, interpreting $\square$ as topological interior. The resulting modal logic, S4, can thus be seen as the modal logic of topological spaces.

Topology provides a minimal environment in which to study continuous functions. Thus we might want a modal logic, not only of topological spaces, but of continuous functions on topological spaces. Let a dynamic topological system be an ordered pair, $\langle X, f\rangle$, where $X$ is a topological space and $f$ is a continuous

P. Kremer $(\bowtie)$

Department of Philosophy, University of Toronto, Toronto, Canada

e-mail:kremer@utsc.utoronto.ca 
function on $X$. We extend the language $\mathcal{L}^{\square}$ to a bimodal language $\mathcal{L}$ by adding the temporal modal operator $\bigcirc$ : we will use $f$ to interpret $\bigcirc$.

Artemov et al. [1] prove the soundness and completeness of a very natural logic S4C, whose topological fragment is just S4, with respect to the class of all dynamic topological systems (See also [2,5]). Thus S4C can be seen as the modal logic of continuous functions on topological spaces. The current paper's main theorem is that $\mathrm{S} 4 \mathrm{C}$ is complete with respect to a particular topological space, namely Cantor space. Thus S4C can also be seen as the modal logic of continuous functions on Cantor space.

The main theorem was first proved by Zhang and Mints [19] - see also Mints and Zhang [11]. The current proof results from an attempt to understand the Zhang-Mints Theorem by proving it independently. It shares some of their central ideas, but proceeds quite differently, and perhaps more simply. (I thank Grigori Mints who got me interested in the problem, and showed me a draft of Zhang and Mints [19].)

To make things precise, a dynamic topological model is an ordered triple $M=$ $\langle X, f, V\rangle$ where $\langle X, f\rangle$ is a dynamic topological system and $V: P V \rightarrow \mathcal{P}(X)$ is a valuation function assigning a subset of $X$ to each propositional variable. The valuation $V$ is extended to all formulas as follows:

$$
\begin{aligned}
V(A \vee B) & =V(A) \cup V(B), \\
V(A \& B) & =V(A) \cap V(B), \\
V(\neg A) & =X-V(A), \\
V(\bigcirc A) & =f^{-1}(V(A)), \quad \text { and } \\
V(\square A) & =\operatorname{Int}(V(A))=\text { the topological interior of } V(A) .
\end{aligned}
$$

A formula $A$ is validated by $M=\langle X, f, V\rangle(M \models A)$ iff $V(A)=X$. $A$ is validated by $D=\langle X, f\rangle(D \models A)$ iff $A$ is validated by $\langle X, f, V\rangle$ for every $V: P V \rightarrow \mathcal{P}(X) . A$ is validated by the topological space $X(X \models A)$ iff $A$ is validated by $\langle X, f\rangle$ for every continuous function $f . A$ is valid $(\models A)$ iff $M \models A$ for every dynamic topological model $M$.

The logic S4C is the logic determined by the axioms of S4 for $\square$, plus

$$
\begin{aligned}
\bigcirc(A \& B) & \equiv(\bigcirc A \& \bigcirc B) \\
\bigcirc(A \vee B) & \equiv(\bigcirc A \vee \bigcirc B) \\
\bigcirc \neg A & \equiv \neg \bigcirc A \\
\bigcirc \square A & \supset \square \bigcirc A
\end{aligned}
$$

and the rules of Modus Ponens, and necessitation for $\square$ and $\bigcirc$. Artemov et al. [1] prove the following soundness and completeness theorem:

Theorem 1.1 $\models A$ iff $A \in \mathrm{S} 4 \mathrm{C}$.

To state our main theorem, we define both Cantor space and a number of related spaces based on trees. 
Let $* \omega$ be the set of finite sequences of natural numbers, including the empty sequence $\Lambda$. We use bold $\mathbf{x}, \mathbf{y}, \mathbf{z}, \mathbf{v}$ to range over ${ }^{*} \omega$. For $\mathbf{x}, \mathbf{y} \in{ }^{*} \omega$, we write $\mathbf{x y}$ or $\mathbf{x}^{\frown} \mathbf{y}$ for $\mathbf{x}$ concatenated with $\mathbf{y}$. For $\mathbf{x}, \mathbf{y} \in{ }^{*} \omega$ we say that $\mathbf{x} \leq \mathbf{y}$ iff $\mathbf{x}$ is an initial segment of $\mathbf{y}$, and $\mathbf{x}<\mathbf{y}$ iff $\mathbf{x} \leq \mathbf{y}$ and $\mathbf{x} \neq \mathbf{y}$. For $\mathbf{x} \in{ }^{*} \omega$ and $k \in \omega$, we write $\mathbf{x} k$ or $\mathbf{x}\urcorner k$ for $\mathbf{x}$ concatenated with $k$. For $\mathbf{x} \in{ }^{*} \omega$, length $(\mathbf{x})$ is the length of $\mathbf{x}$; and for $m<$ length $(\mathbf{x}), \mathbf{x}_{m}$ is the $m^{\text {th }}$ member of $\mathbf{x}$. So $\mathbf{x}=\mathbf{x}_{0}, \mathbf{x}_{1}, \ldots, \mathbf{x}_{\text {length }(\mathbf{x})-1}$.

For our purposes, a tree is any $T \subseteq{ }^{*} \omega$ satisfying the following:

$$
\begin{aligned}
& \Lambda \in T ; \\
& \forall \mathbf{x} \in T, \exists k \in \omega, \mathbf{x} k \in T ; \quad \text { and } \\
& \forall \mathbf{x} \in{ }^{*} \omega, \forall k \in \omega, \text { if } \mathbf{x} k \in T \text { then } \mathbf{x} \in T .
\end{aligned}
$$

We can think of the members of $T$ as nodes in $T$. $T$ is nontrivially branching iff

$$
\forall \mathbf{x} \in T, \exists k, j \in \omega, \quad k \neq j \text { and } \mathbf{x} k, \mathbf{x} j \in T .
$$

$T$ is finitely branching iff

$$
\forall \mathbf{x} \in T \text {, the } \operatorname{set}\{k \in \omega: \mathbf{x} k \in T\} \text { is finite. }
$$

We will be interested in paths through trees: these can be represented by members of ${ }^{\omega} \omega$, i.e. by $\omega$-long sequences of natural numbers. We use bold-italic $\boldsymbol{x}, \boldsymbol{y}, \boldsymbol{z}, \boldsymbol{v}$ to range over ${ }^{\omega} \omega$. For $\mathbf{x} \in{ }^{*} \omega$ and $\boldsymbol{y} \in{ }^{\omega} \omega$, we write $\mathbf{x y}$ or $\mathbf{x} \frown \boldsymbol{y}$ for $\mathbf{x}$ concatenated with $\boldsymbol{y}$. For $\boldsymbol{x} \in{ }^{\omega} \omega$ and $m \in \omega, \boldsymbol{x}_{m}$ is the $m$ th member of $\boldsymbol{x}$. So $\boldsymbol{x}=\boldsymbol{x}_{0}, \boldsymbol{x}_{1}, \ldots, \boldsymbol{x}_{m}, \ldots$. And for $\boldsymbol{x} \in{ }^{\omega} \omega$ and $m \in \omega, \boldsymbol{x}\lceil m$ is the finite sequence consisting of the first $m$ member of $\boldsymbol{x}$. So $\boldsymbol{x}\left\lceil m=\boldsymbol{x}_{0}, \boldsymbol{x}_{1}, \ldots, \boldsymbol{x}_{m-1}\right.$. If $T$ is a tree, a $T$-path is an $\boldsymbol{x} \in{ }^{\omega} \omega$ such that $\boldsymbol{x} \backslash m \in T$ for every $m \in \omega$. For every tree $T$ we define

$$
\operatorname{path}(T)=\{\boldsymbol{x}: \boldsymbol{x} \text { is a } T \text {-path }\}
$$

A $T$-path through the node $\mathbf{x} \in T$ is any $T$-path of the form $\mathbf{x}^{\frown} \boldsymbol{y}$.

We impose a topology on $\operatorname{path}(T)$ as follows. For $\mathbf{x} \in T$, let

$\mathbf{B}_{\mathbf{x}}^{T}=\left\{\mathbf{x}^{\frown} \boldsymbol{y}: \boldsymbol{y} \in{ }^{\omega} \omega\right.$ and $\left.\mathbf{x}^{\frown} \boldsymbol{y} \in \operatorname{path}(T)\right\}=$ the set of $T$-paths through the node $\mathbf{x}$.

Generalized T-Cantor Space is path $(T)$ with the topology determined by the basis sets $\mathbf{B}_{\mathbf{x}}^{T}$, where $\mathbf{x} \in T$. And Cantor Space is $\mathcal{C}=$ path $\left.^{*} 2\right)$, where ${ }^{*} 2$ is the set of finite sequences of 0 and 1 . Our main theorem is as follows.

Theorem 1.2 (Soundness and completeness in Cantor space) $\mathcal{C} \models A$ iff $A \in$ S4C.

Since soundness (i.e. the $\Leftarrow$ direction of the 'iff') is routine, we concentrate on completeness: if $\mathcal{C} \models A$ then $A \in \mathrm{S} 4 \mathrm{C}$. Or, as we will prove it, if $A \notin \mathrm{S} 4 \mathrm{C}$ 
then $\mathcal{C} \not \forall A$ (see Sects. $2-5)$. First, we reduce the problem of showing completeness in $\mathcal{C}$ to the problem of showing completeness in path $(T)$, where $T$ is any nontrivially finitely branching tree.

Lemma 1.3 Suppose that $T$ is a nontrivially finitely branching tree. Then, for every formula $A$, we have $\mathcal{C} \models A$ iff path $(T) \models A$.

Proof It suffices to construct a one-one onto continuous open function, $\phi$ : $\operatorname{path}(T) \rightarrow \mathcal{C}$. First we construct a one-one function $\psi: T \rightarrow * 2$. Let

$$
\psi(\Lambda)=\Lambda
$$

Suppose that $\psi(\mathbf{x})$ is defined for $\mathbf{x} \in T$, and let $k_{0}, \ldots, k_{m-1}$ be an exhaustive strictly increasing list of natural numbers such that $\mathbf{x} k_{n} \in T$ for each $n<m$. Note that $m$ is finite since $T$ is finitely branching, and that $m>1$ since $T$ is nontrivially branching. If $m=5$ then we have the following picture of a portion of the tree $T$ :

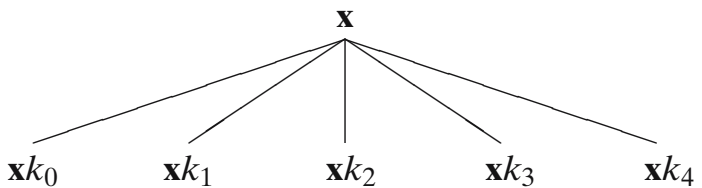

We define $\psi\left(\mathbf{x} k_{n}\right)$ as follows, for each $n<m$ :

$$
\begin{aligned}
& \psi\left(\mathbf{x} k_{n}\right)=\psi(\mathbf{x}) \frown 1^{n} \frown 0 \text { if } n<m-1, \\
& \psi(\mathbf{x}) \frown 1^{n} \text { if } n=m-1 .
\end{aligned}
$$

In this definition, $1^{n}$ is the finite sequence consisting of $n$ occurrences of 1 . The portion of $T$ pictured above would get mapped by $\psi$ into a portion of *2 as follows:

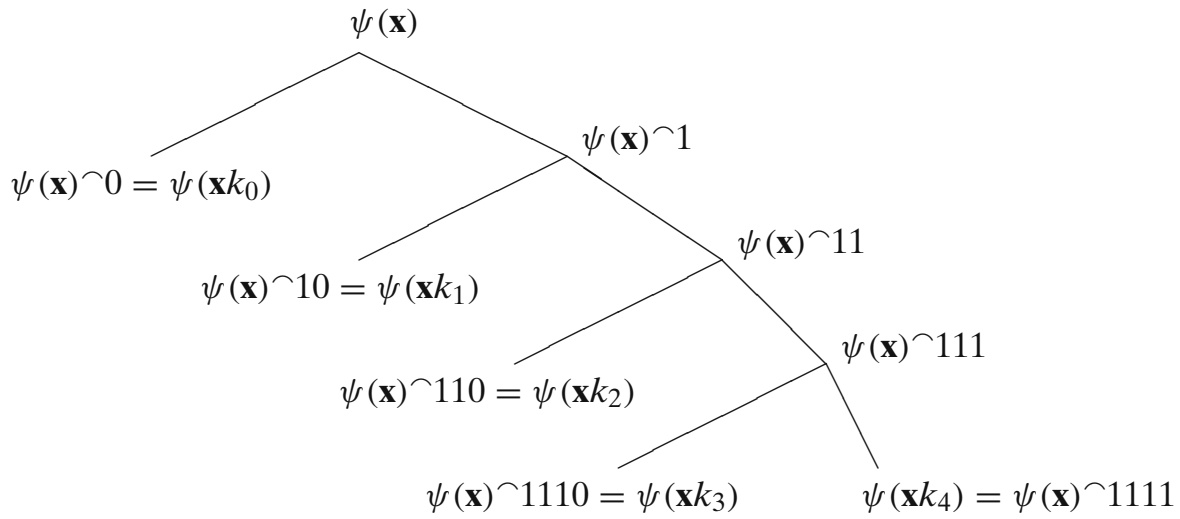


The function $\psi: T \rightarrow^{*} 2$ induces a function $\phi: \operatorname{path}(T) \rightarrow \mathcal{C}$ as follows: $\phi(x)$ is the unique *2-path through the nodes $\psi(\boldsymbol{x}\lceil 0), \psi(\boldsymbol{x}\lceil 1), \psi(\boldsymbol{x}\lceil 2), \psi(\boldsymbol{x}\lceil 3), \ldots$. Note that $\phi$ is, as desired, one-one, onto, continuous and open.

\section{Dynamic Kripke models}

Our proof of completeness of $\mathrm{S} 4 \mathrm{C}$ in $\mathcal{C}$ relies on an alternate, Kripke-style, semantics for S4C. A dynamic Kripke frame is an ordered triple $K=\langle W, R, f\rangle$ where $W$ is a non-empty set (of worlds), $R$ is a reflexive and transitive relation on $W$, and $f$ is a function on $W$ which is $R$-monotonic with respect to $R$, i.e. $w R w^{\prime} \Rightarrow f w R f w^{\prime}$. World $w^{\prime}$ is a successor of world $w$ iff $w R w^{\prime} . w$ is $R$-equivalent to $w^{\prime}$ (in symbols, $w \equiv_{R} w^{\prime}$ ) iff $w R w^{\prime}$ and $w^{\prime} R w . r \in W$ is a root world of $\langle W, R, f\rangle$ iff both $f r=r$ and $\forall w \in W, r R w .\langle W, R, f\rangle$ is rooted iff there is some root world $r \in W$. We define the interior of $X \subseteq W$ :

$$
\operatorname{Int}(X)=_{d f}\left\{w \in W: \forall w^{\prime} \in W \text {, if } w R w^{\prime} \text { then } w^{\prime} \in X\right\}
$$

A dynamic Kripke model is a quartuple $M=\langle W, R, f, V\rangle$, where $\langle W, R, f\rangle$ is a dynamic Kripke frame and $V$ is a valuation function assigning a subset of $W$ to each propositional variable. The valuation $V$ is extended to all formulas as follows:

$$
\begin{aligned}
V(A \vee B) & =V(A) \cup V(B) \\
V(A \& B) & =V(A) \cap V(B) \\
V(\neg A) & =X-V(A) \\
V(\bigcirc A) & =f^{-1}(V(A)) \\
V(\square A) & =\operatorname{Int}(V(A)) .
\end{aligned}
$$

$\langle W, R, f, V\rangle$ is rooted iff $\langle W, R, f\rangle$ is rooted. A formula $A$ is validated by $M=\langle W, R, f, V\rangle$ iff $V(A)=W$. Notation: $M \models A$.

Artemov et al. [1] prove soundness and completeness in dynamic Kripke models:

Theorem 2.1 $A \in \mathrm{S} 4 \mathrm{C}$ iff $A$ is validated by every dynamic Kripke model.

Artemov et al. [1] also prove that S4C has the finite model property:

Theorem 2.2 $A \in \mathrm{S} 4 \mathrm{C}$ iff $A$ is validated by every rooted finite dynamic Kripke model.

\section{Constructing a labelled tree}

Given Lemma 1.3 and Theorem 2.2, proving our main theorem, Theorem 1.2, reduces to proving the following: 
Theorem 3.1 If the formula $Z$ is invalidated by some rooted finite dynamic Kripke model, then path $(T) \forall \forall Z$ for some nontrivially finitely branching tree $T$.

Sections 3, 4 and 5 are devoted to proving Theorem 3.1. Suppose that the formula $Z$ is invalidated by some rooted finite dynamic Kripke model $M=$ $\langle W, R, f, V\rangle$ with root $r$. We will construct a nontrivially finitely branching tree $T$ such that $\operatorname{path}(T) \not \models Z$.

A labelled tree is an ordered pair $\boldsymbol{T}=\langle T, L\rangle$ where $T$ is a tree and $L: T \rightarrow W$. $L$ can be thought of as a function that labels nodes in $T$ with worlds from $W$. We say that $\boldsymbol{T}=\langle T, L\rangle$ is nontrivially [finitely] branching iff $T$ is nontrivially [finitely] branching. Given a labelled tree $\boldsymbol{T}=\langle T, L\rangle$, we define the labelled tree $f(\boldsymbol{T})=\langle T, f \circ L\rangle$ where $f \circ L(\mathbf{x})=f(L(\mathbf{x}))$. The new labelled tree $f(\boldsymbol{T})$ is a relabelling of the underlying tree $T$ : if the node $\mathbf{x}$ is labelled with the world $w$ in $\boldsymbol{T}$, then the same node $\mathbf{x}$ is labelled with the world $f(w)$ in $f(\boldsymbol{T})$. A labelled tree $\boldsymbol{T}=\langle T, L\rangle$ is consistent iff

(i) $L(\Lambda)=r$; and

(ii) $L(\boldsymbol{x}) R L(\mathbf{x} k)$, whenever both $\mathbf{x}$ and $\mathbf{x} k \in T$.

Note the following:

(3.1) If the labelled tree $\boldsymbol{T}$ is consistent, then so is $f(\boldsymbol{T})$. (This follows from the $R$-monotonicity of $f$.)

Given $\boldsymbol{T}=\langle T, L\rangle$ and $\boldsymbol{T}^{\prime}=\left\langle T^{\prime}, L^{\prime}\right\rangle$, we say that $\boldsymbol{T} \leq \boldsymbol{T}^{\prime}$ iff $T \subseteq T^{\prime}$ and $L(\mathbf{x})=L^{\prime}(\mathbf{x})$, for every $\mathbf{x} \in T$. A labelled tree $\boldsymbol{T}=\langle T, L\rangle$ is complete iff, in addition to (i) and (ii), we also have

(iii) $\forall \mathbf{x} \in T, \forall w \in W$, if $L(\mathbf{x}) R w$ then $\exists k \in \omega, \mathbf{x}^{\urcorner} k \in T$ and $L\left(\mathbf{x}^{\frown} k\right)=w$.

Note the following:

(3.2) If $\boldsymbol{T}$ is a consistent nontrivially finitely branching labelled tree, then there is a complete nontrivially finitely branching labelled tree $\boldsymbol{T}^{\prime}$ such that $\boldsymbol{T} \leq \boldsymbol{T}^{\prime}$. We say that such a $\boldsymbol{T}^{\prime}$ is a completion of $\boldsymbol{T}$.

We will soon define a sequence of complete nontrivially finitely branching labelled trees,

$$
\boldsymbol{T}_{0}, \boldsymbol{T}_{1}, \boldsymbol{T}_{2}, \boldsymbol{T}_{3}, \ldots
$$

Then we will define a great big tree $\boldsymbol{T}$ by gluing $\boldsymbol{T}_{0}, \boldsymbol{T}_{1}, \boldsymbol{T}_{2}, \boldsymbol{T}_{3}, \ldots$ together as follows: 


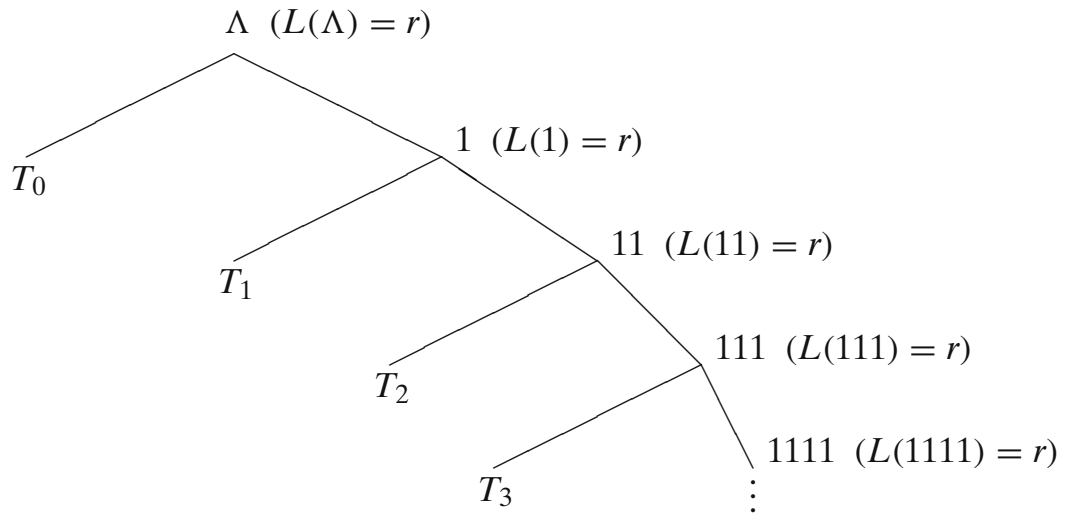

The point will be clearer when we give the formal definition of $\boldsymbol{T}$, below. As it turns out, $\boldsymbol{T}$ will be nontrivially finitely branching and consistent, but not (quite) complete.

To define $\boldsymbol{T}_{0}$, let $n$ be any number $\geq 2$ and $\geq$ the cardinality of $W$. Let $T_{0}={ }^{*} n=$ the set of finite sequences of members of $\{0, \ldots, n-1\}$. For each $w \in W$, let $\operatorname{succ}(w, 0), \operatorname{succ}(w, 1), \ldots, \operatorname{succ}(w, n-1)$ be a possibly repeating list of all the successors of $w$. Now define $L_{0}(\mathbf{x})$, for $\mathbf{x} \in T_{0}={ }^{*} n$, inductively as follows:

$$
\begin{aligned}
L_{0}(\Lambda) & =r \\
L_{0}\left(\mathbf{x}^{\frown} k\right) & =\operatorname{succ}(L(\mathbf{x}), k) .
\end{aligned}
$$

Let $\boldsymbol{T}_{0}=\left\langle T_{0}, L_{0}\right\rangle$. Note that $\boldsymbol{T}_{0}$ is nontrivially finitely branching and complete. Suppose that the complete nontrivially finitely branching labelled tree $\boldsymbol{T}_{k}=\left\langle T_{k}, L_{k}\right\rangle$ has been defined. Then let $\boldsymbol{T}_{k+1}=\left\langle T_{k+1}, L_{k+1}\right\rangle$ be any completion of $f\left(\boldsymbol{T}_{k}\right)$. We know that a completion of $f\left(\boldsymbol{T}_{k}\right)$ exists, by (3.1) and (3.2), above. This gives us our desired sequence of complete nontrivially finitely branching labelled trees, $\boldsymbol{T}_{0}, \boldsymbol{T}_{1}, \boldsymbol{T}_{2}, \boldsymbol{T}_{3}, \ldots$. Note also that $T_{0} \subseteq T_{1} \subseteq T_{2} \subseteq$ $T_{3} \subseteq \cdots$, and that $L_{k+1}(\mathbf{x})=f\left(L_{k}(\mathbf{x})\right)$, for each $\mathbf{x} \in T_{k}$.

Now we define our great big labelled tree $\boldsymbol{T}=\langle T, L\rangle$. Recall the picture above, indicating how we are to build $\boldsymbol{T}$. In the following definitions, $1^{k}$ is the finite sequence consisting of $k$ occurrences of 1 :

$$
\begin{aligned}
& T=\left\{1^{k \frown 0 \frown} \mathbf{x}: k \in \omega \text { and } \mathbf{x} \in T_{k}\right\} \cup\left\{1^{k}: k \in \omega\right\}, \\
& L\left(1^{k}\right)=r \text {, for each } k \in \omega \text {, and }
\end{aligned}
$$

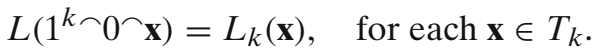

Note that $\boldsymbol{T}$ is nontrivially finitely branching and consistent. $\boldsymbol{T}$ might not be complete. We do however have the following, which makes $\boldsymbol{T}$ close enough to complete for our purposes: 
(3.3) If $\mathbf{y} \in T$ and $L(\mathbf{y}) R w$, then $\exists \boldsymbol{z} \in \operatorname{path}(T)$ and $\exists k \in \omega$ such that $\mathbf{y}$ is an inital segment of $\boldsymbol{z}$, and $\forall m \geq k, L(\boldsymbol{z} \mid m)=w$.

Finally, notice that

$$
L(1 \frown \mathbf{x})=f(L(\mathbf{x})), \quad \text { for each } \mathbf{x} \in T .
$$

Anticipating Sect. 5, we note that the dynamic topological system of interest to us will be $\langle\operatorname{path}(T), g\rangle$, where $g(\boldsymbol{x})=1 \frown \boldsymbol{x}$, for each $\boldsymbol{x} \in \operatorname{path}(T)$.

\section{Sequences of worlds}

An $\omega$-long sequence $w_{0}, w_{1}, w_{2}, w_{3}, w_{4}, \ldots, w_{m}, \ldots$, of worlds is ascending iff $w_{i} R w_{j}$ whenever $i \leq j$. An $\omega$-long sequence $w_{0}, w_{1}, w_{2}, w_{3}, w_{4}, \ldots, w_{m}, \ldots$, of worlds settles at the world $w$ iff $\exists i, \forall j \geq i, w_{j} \equiv_{R} w$. Since $W$ is finite, every ascending $\omega$-long sequence of worlds settles at some world, though some ascending $\omega$-long sequence of worlds might settle at more than one world. Note:

(4.1) If some ascending $\omega$-long sequence of worlds settles at $w$ and at $w^{\prime}$, then $w \equiv \equiv_{R} w^{\prime}$.

(4.2) If $w$ is a member of an ascending $\omega$-long sequence of worlds that settles at $w^{\prime}$, then $w R w^{\prime}$.

We associate with every $\boldsymbol{x} \in \operatorname{path}(T)$, a sequence of worlds $\operatorname{seq}(\boldsymbol{x})$ :

$$
\operatorname{seq}(\boldsymbol{x}){ }_{d f} L(\boldsymbol{x}\lceil 0), L(\boldsymbol{x}\lceil 1), L(\boldsymbol{x}\lceil 2), L(\boldsymbol{x}\lceil 3), L(\boldsymbol{x}\lceil 4), \ldots
$$

Note the following:

(4.3) $\operatorname{seq}(\boldsymbol{x})$ is always ascending since $\boldsymbol{T}$ is consistent, and

(4.4) If $\operatorname{seq}(\boldsymbol{x})=w_{0}, w_{1}, w_{2}, w_{3}, w_{4}, \ldots, w_{m}, \ldots$ then $\operatorname{seq}(1 \frown \boldsymbol{x})=r, f w_{0}, f w_{1}$, $f w_{2}, f w_{3}, f w_{4}, \ldots, f w_{m}, \ldots$.

We say that $\boldsymbol{x}$ settles at $w$ iff $\operatorname{seq}(\boldsymbol{x})$ settles at $w$. Let sett be a function assigning, to each ascending sequence of worlds, a world at which the sequence settles: such a function exists since $W$ is finite. Note the following:

(4.5) If $\operatorname{seq}(\boldsymbol{x})$ settles at $w$ then $w \equiv_{R} \operatorname{sett}(\operatorname{seq}(\boldsymbol{x}))$.

(4.6) $\operatorname{sett}(\operatorname{seq}(1 \frown \boldsymbol{x})) \equiv_{R} f(\operatorname{sett}(\operatorname{seq}(\boldsymbol{x}))$.

Finally, we define a function world $: \operatorname{path}(T) \rightarrow W$. If $\boldsymbol{x} \in \operatorname{path}(T)$, say that $\boldsymbol{x}$ is a bootstrapper iff

$$
\text { either } \boldsymbol{x}=1^{\omega} \text { or } \boldsymbol{x} \neq 1 \frown \boldsymbol{y} \text {, for any } \boldsymbol{y} \in \operatorname{path}(T) \text {. }
$$

In this definition, $1^{\omega}$ is the infinite sequence of 1 's: $11111 \ldots$. Note the following:

(4.7) For every $\boldsymbol{x} \in \operatorname{path}(T)-\left\{1^{\omega}\right\}$, there is a unique bootstrapper $\boldsymbol{y} \in \operatorname{path}(T)$ and a unique $k \in \omega$, such that $\boldsymbol{x}=1^{k \frown y}$. (Proof: If $\boldsymbol{x} \neq 1^{\omega}$, then just keep removing occurrences of 1 from the beginning of $\boldsymbol{x}$ until you are 
reduced to a bootstrapper. This will give you the unique bootstrapper $y$ and the unique $k \in \omega$. It is worth noting that this claim fails for $\boldsymbol{x}=1^{\omega}$, since $1^{\omega}=1^{k \frown} 1^{\omega}$, for every $k \in \omega$.)

So we define the function world as follows:

if $\boldsymbol{x}=1^{\omega}$, then $\operatorname{world}(\boldsymbol{x})=r$, the root world; and

if $\boldsymbol{x} \neq 1^{\omega}$, then $\operatorname{world}(\boldsymbol{x})=f^{k}(\operatorname{sett}(\operatorname{seq}(\boldsymbol{y})))$, where $\boldsymbol{y}$ is a bootstrapper, and $\boldsymbol{x}=1^{k \frown \boldsymbol{y}}$.

To see how this works, note that for each bootstrapper $\boldsymbol{y} \in \operatorname{path}(T)$, we have

$$
\begin{aligned}
& \operatorname{world}(\boldsymbol{y})=\operatorname{sett}(\operatorname{seq}(\boldsymbol{y})) \\
& \operatorname{world}(1 \frown \boldsymbol{y})=f(\operatorname{sett}(\operatorname{seq}(\boldsymbol{y}))) \\
& \text { world }(11 \frown \boldsymbol{y})=f^{2}(\operatorname{sett}(\operatorname{seq}(\boldsymbol{y}))) \\
& \text { world }(111 \frown \boldsymbol{y})=f^{3}(\operatorname{sett}(\operatorname{seq}(\boldsymbol{y}))) \\
& \text { and so on. }
\end{aligned}
$$

The above even holds for $\boldsymbol{y}=1^{\omega}: \operatorname{world}\left(1^{k} \boldsymbol{y}\right)=r=f^{k}\left(\operatorname{sett}\left(\operatorname{seq}\left(1^{\omega}\right)\right)\right)$, for every $k \in \omega$, since $\operatorname{seq}\left(1^{\omega}\right)=r, r, r, r, \ldots$. Now notice the following, for each $\boldsymbol{x} \in \operatorname{path}(T):$

(4.8) $\operatorname{world}(\boldsymbol{x}) \equiv_{R} \operatorname{sett}(\operatorname{seq}(\boldsymbol{x}))$

(4.9) $\operatorname{seq}(\boldsymbol{x})$ settles at $\operatorname{world}(\boldsymbol{x})$

(4.10) $\forall w \in W, \exists \boldsymbol{x} \in \operatorname{path}(T), \operatorname{world}(\boldsymbol{x})=w$.

(4.11) $\operatorname{world}(1 \frown \boldsymbol{x})=f(\operatorname{world}(\boldsymbol{x}))$.

\section{A dynamic topological model}

We define the dynamic topological model $M^{\prime}=_{d f}\left\langle\operatorname{path}(T), g, V^{\prime}\right\rangle$, where

$$
\begin{aligned}
g(\boldsymbol{x}) & =1^{\frown \boldsymbol{x}} \text {, and } \\
V^{\prime}(p) & =\{\boldsymbol{x} \in \operatorname{path}(T): \operatorname{world}(\boldsymbol{x}) \in V(p)\} .
\end{aligned}
$$

Note that $g$ is continuous on path $(T)$. We now show by induction that, for every formula $A$,

(5.1) $\forall \boldsymbol{x} \in \operatorname{path}(T), \boldsymbol{x} \in V^{\prime}(A)$ iff $\operatorname{world}(\boldsymbol{x}) \in V(A)$.

If $A$ is atomic, the result is given by definition. If $A$ is of the form $\neg B$, or $(B \& C)$, or $(B \vee C)$, then the inductive step is straightforward.

Induction step, $\square$ : Suppose that $A$ is of the form $\square B$. Fix $\boldsymbol{x} \in \operatorname{path}(T)$. We will show both directions of the desired biconditional separately. $(\Rightarrow)$ Assume that $\boldsymbol{x} \in V^{\prime}(A)$. Then $\boldsymbol{x} \in \operatorname{Int}\left(V^{\prime}(B)\right)$. So there is some basis set $\mathbf{B}_{\mathbf{y}}^{T}$, with $\mathbf{y} \in T$, such that $\boldsymbol{x} \in \mathbf{B}_{\mathbf{y}}^{T} \subseteq V^{\prime}(B)$. Note right away that $L(\mathbf{y}) R \operatorname{world}(\boldsymbol{x})$. (Proof: Since $\mathbf{y}$ is an initial segment of $\boldsymbol{x}, L(\mathbf{y})$ is one of the worlds in $\operatorname{seq}(\boldsymbol{x})$. So $L(\mathbf{y}) R$ world $(\boldsymbol{x})$ follows from (4.2) and the choice of sett.) To see that $\operatorname{world}(\boldsymbol{x}) \in V(\square B)=$ $\operatorname{Int}(V(B))$, suppose that $\operatorname{world}(\boldsymbol{x}) R w$. It suffices to show that $w \in V(B)$. Since $L(\mathbf{y}) R$ world $(\boldsymbol{x})$ and $\operatorname{world}(\boldsymbol{x}) R w$, we have $L(\mathbf{y}) R w$. So by (3.3), $\exists \boldsymbol{z} \in \operatorname{path}(T)$ 
such that $\mathbf{y}$ is an inital segment of $\boldsymbol{z}$, and $\exists k \in \omega, \forall m \geq k, L(\boldsymbol{z} \mid m)=w$. Thus $\operatorname{seq}(\boldsymbol{z})$ settles at exactly one world, namely $w$. So by $(4.9), \operatorname{world}(\boldsymbol{z})=w$. Since $\mathbf{y}$ is an initial segment of $z$, we have $z \in \mathbf{B}_{\mathbf{y}}^{T}$. So $z \in V^{\prime}(B)$. So by the inductive hypothesis, $w=$ world $(\boldsymbol{z}) \in V(B)$, as desired.

$(\Leftarrow)$ Assume that $\operatorname{world}(\boldsymbol{x}) \in V(A)=V(\square B)=\operatorname{Int}(V(B))$. By (4.9) and the definition of $\operatorname{seq}(\boldsymbol{x})$, there is a $k \in \omega$, such that $L(\boldsymbol{x} \mid m) \equiv_{R} \operatorname{world}(\boldsymbol{x})$, for every $m \geq k$. Let $\mathbf{y}=\boldsymbol{x} \backslash k$. Note that $\boldsymbol{x} \in \mathbf{B}_{\mathbf{y}}^{T}$. So it suffices to show that $\mathbf{B}_{\mathbf{y}}^{T} \subseteq V^{\prime}(B)$. So suppose that $\boldsymbol{z} \in \mathbf{B}_{\mathbf{y}}^{T}$. Since $\boldsymbol{z}\lceil k=\mathbf{y}$ and since the labelled tree $\boldsymbol{T}$ is consistent, we have $L(\mathbf{y}) R L(\boldsymbol{z} \mid m)$, for every $m \geq k$. So $L(\mathbf{y}) R w$, for every world w at which $\boldsymbol{z}$ settles. So $L(y) R \operatorname{world}(\boldsymbol{z})$. Since $L(\mathbf{y}) \equiv_{R} \operatorname{world}(\boldsymbol{x})$, we have $\operatorname{world}(\boldsymbol{x}) R$ world $(\boldsymbol{z})$. Thus $\operatorname{world}(\boldsymbol{z}) \in V(B)$. So, by the inductive hypothesis, $z \in V^{\prime}(B)$, as desired.

Induction step, $\bigcirc$ : Suppose that $A$ is of the form $\bigcirc B$. Fix $\boldsymbol{x} \in \operatorname{path}(T)$. Note: $\boldsymbol{x} \in V^{\prime}(A)$ iff $\boldsymbol{x} \in V^{\prime}(\bigcirc B)$ iff $g \boldsymbol{x} \in V^{\prime}(B)$ iff $\operatorname{world}(g \boldsymbol{x}) \in V(B)$ (by the inductive hypothesis) iff $f(\operatorname{world}(\boldsymbol{x})) \in V(B)$ (by (4.11) and the definition of $g$ ) iff $\operatorname{world}(\boldsymbol{x}) \in V(\bigcirc B)$ iff $\operatorname{world}(\boldsymbol{x}) \in V(A)$.

This completes the proof of (5.1). To see that $\operatorname{path}(T) \not \models Z$ it suffices to show that $M^{\prime} \not \models Z$. Recall that $M \not \models Z$ (see the beginning of Sect. 3). Choose a world $w \in W$ such that $w \notin V(Z)$. Now choose a $\boldsymbol{x} \in \operatorname{path}(T)$ such that world $(\boldsymbol{x})=w$ [See (4.10)]. Then, by (5.1), $\boldsymbol{x} \notin V^{\prime}(Z)$. So $M^{\prime} \not \models Z$, as desired. This completes the proof of the main theorem.

\section{Related issues}

\subsection{The modal logic of continuous functions on $\mathbb{R}$}

McKinsey and Tarski [10] show that S4 is not only the modal logic of topological spaces, but also the modal logic of a host of particular topological spaces, among them Cantor space and $\mathbb{R}$. Similarly, S4C is the not only modal logic of continuous functions on topological spaces, but also of continuous functions on a particular topological space, Cantor space. We might conjecture that, by analogy with the McKinsey-Tarski result, S4C is also the modal logic of continuous functions on $\mathbb{R}$.

But it isn't. Consider the formula,

$$
A={ }_{d f}(\square \bigcirc p \supset \bigcirc \diamond \square p) \vee(\bigcirc q \supset \square \bigcirc q) .
$$

(In general, $\diamond B$ is defined as $\neg \square \neg B$. Thus, if $\langle X, f, V\rangle$ is a dynamic topological model, then $V(\diamond B)=C l(V(B))$, where $C l$ is topological closure.) We will see that $A$ is a counterexample to the claim that if $\mathbb{R}=A$ then $A \in S 4 C$. (The formula $A$ is a simplification of a formula of van Benthem's, which is itself a simplification of a formula of the author's. This counterexample is also given in Kremer and Mints [5]. The incompleteness of S4C in $\mathbb{R}$ was discovered independently by Slavnov [16], who uses a different counterexample.)

First, $A \notin \mathrm{S} 4 \mathrm{C}$. To see this, consider the dynamic topological model $M=$ $\langle X, f, V\rangle$, where 


$$
\begin{aligned}
X & =\{0,1,2\} \text { with three open sets: } \emptyset,\{0\}, \text { and }\{0,1,2\} ; \\
f 0 & =1 \text { and } f 1=2 \text { and } f 2=1 ; \text { and } \\
V(p) & =\{1,2\} \text { and } V(q)=\{2\} .
\end{aligned}
$$

Note: $V(\bigcirc p)=\{0,1,2\}$. So $V(\square \bigcirc p)=\{0,1,2\}$. Meanwhile $V(\square p)=\emptyset$. So $V(\bigcirc \diamond \square p)=\emptyset$. Thus $V(\square \bigcirc p \supset \bigcirc \diamond \square p)=\emptyset$. Also, $V(\bigcirc q)=\{1\}$. So $V(\square \bigcirc q)=$ $\emptyset$. So $V(\bigcirc q \supset \square \bigcirc q)=\{0,2\}$. Putting this all together, $V(A)=\{0,2\} \neq X$. So $M \not \models A$. So $A \notin \mathrm{S} 4 \mathrm{C}$.

But $\mathbb{R} \models A$. To see this, suppose that $M^{\prime}=\left\langle\mathbb{R}, f^{\prime}, V^{\prime}\right\rangle$ is a dynamic topological model such that $M^{\prime} \not \models A$. Choose $x \in \mathbb{R}$ such that $x \notin V^{\prime}(A)$. So

(6.1) $x \in V^{\prime}(\square \bigcirc p)$; so there is an open interval $I$ such that $x \in I \subseteq V^{\prime}(\square \bigcirc p)$; so $f^{\prime} x \in f^{\prime}(I) \subseteq V^{\prime}(p)$;

(6.2) $x \notin V^{\prime}(\bigcirc \diamond \square p)$; so $f^{\prime} x \notin V(\diamond \square p)$; so $f^{\prime} x \notin C l\left(\operatorname{Int}\left(V^{\prime}(p)\right)\right.$;

(6.3) $x \in V^{\prime}(\bigcirc q)$; so $f^{\prime} x \in V^{\prime}(q)$; and

(6.4) $x \notin V^{\prime}(\square \bigcirc q)$; so $y \notin V^{\prime}(\bigcirc q)$ for some $y \in I$; so $f^{\prime} y \notin V^{\prime}(q)$; so $f^{\prime} y \neq f^{\prime} x$; so $I$ is not a singleton set.

Since $f^{\prime}$ is continuous, $I$ is an open interval, and $f^{\prime}(I)$ is not a singleton set, $f^{\prime}(I)$ is either an open interval, a closed interval, or a semiclosed interval. (A semiclosed interval is an interval of the form $(a, b]$ or $[a, b)$, where $a<b$.) In any case, $f^{\prime}(I) \subseteq C l\left(\operatorname{Int}\left(f^{\prime}(I)\right)\right.$. So $f^{\prime} x \in C l\left(\operatorname{Int}\left(f^{\prime}(I)\right)\right.$. Also, $f^{\prime}(I) \subseteq V^{\prime}(p)$ by (6.1). So $f^{\prime} x \in C l\left(\operatorname{Int}\left(f^{\prime}(I)\right) \subseteq C l\left(\operatorname{Int}\left(V^{\prime}(p)\right)\right.\right.$. But this contradicts (6.2).

Thus, $\mathrm{S} 4 \mathrm{C}$ is not the modal logic of continuous functions on $\mathbb{R}$, i.e. $\mathrm{S} 4 \mathrm{C} \neq$ $\mathrm{S} 4 \mathbb{R}$ where $\mathrm{S} 4 \mathbb{R}={ }_{d f}\{B: \mathbb{R} \models B\}$. We do not know whether $\mathrm{S} 4 \mathbb{R}$ is axiomatizable, but we conjecture that it is, and, moreover, that $\mathrm{S} 4 \mathbb{R}=\mathrm{S} 4 \mathrm{C}+(\square \bigcirc p \supset$ $\bigcirc \diamond \square p) \vee(\bigcirc q \supset \square \bigcirc q)$, or something close to that.

\subsection{Dynamic topological logic}

The purely temporal fragment of $\mathrm{S} 4 \mathrm{C}$ can be axiomatized with any axioms for classical logic, together with $\bigcirc(A \& B) \equiv(\bigcirc A \& \bigcirc B), \bigcirc(A \vee B) \equiv(\bigcirc A \vee \bigcirc B)$ and $\bigcirc \neg A \equiv \neg \bigcirc A$; and the rules of Modus Ponens and necessitation for $\bigcirc$. (The continuity of the function $\mathrm{f}$ is irrelevant.) This gives a quick decision procedure for whether or not $A$ is a theorem: push all the occurrences of $\bigcirc$ down into the atomic sentences, and then check whether the result is a theorem of classical logic.

Things get more interesting, on the purely temporal side, if we add another temporal modality $*$, meaning "henceforth": for a dynamic topological model $\langle X, f, V\rangle$, we stipulate that $V(* A)=\cap_{n \geq 0} f^{-n}(P)$. The resulting, purely temporal, logic can be axiomatized by the classical tautologies; $\mathrm{S} 4$ axioms for $*$; $(\bigcirc(A \vee B) \equiv(\bigcirc A \vee \bigcirc B)) ;(\bigcirc \neg A \equiv \neg \bigcirc A) ;(\bigcirc * A \equiv * \bigcirc A) ;(* A \supset \bigcirc A)$; the induction axiom $(A \& *(A \supset \supset A) \supset * A)$; and the rules of Modus Ponens, and necessitation for $*$. (Such a logic was first put forward by von Wright [17, 18] and Prior [12]. Segerberg [14] credits Dana Scott, Hans Kamp and Kit Fine with unpublished axiomatizations and completeness proofs. The first published completeness proof occurs in Segerberg [15] (a Russian translation of 
Segerberg [13], which did not appear in print until 1989). See also Kröger [8] and Goldblatt [3].)

The dynamic topological logic programme of Kremer and Mints [5] considers trimodal interior-next-henceforth logics, and some of their fragments. This programme is closely related to the one introduced independently by Artemov et al. [1]. The current paper is part of the DTL programme, axiomatizing the interior-henceforth fragment of the dynamic topological logic of Cantor space. We note that a large number of logics in the full trimodal language are not axiomatizable: This is a recent result of Konev et al. [4]. A number of questions remain open, some concerning axiomatizations, and others concerning the relationships among DTLs: see Kremer and Mints [5] for some examples.

\section{References}

1. Artemov, S., Davoren, J., Nerode, A.: Modal logics and topological semantics for hybrid systems. Technical Report MSI 97-05, Cornell University, June 1997 (available at http://www.cs.gc.cuny.edu// sartemov/) (1997)

2. Davoren, J.: Modal Logics for Continuous Dynamics. PhD Thesis, Cornell University (1998)

3. Goldblatt, R.: Logics of Time and Computation. 2nd edn. Center for the Study of Language and Information Lecture Notes No. 7. Stanford University Press, Stanford (1992)

4. Konev, B., Kontchakov, R., Tishovsky, D., Wolter, F., Zakharyaschev, M.: The undecidability of dynamic topological logics. manuscript (available at http://www.dcs.kcl.ac.uk/staff/mz/) (2004)

5. Kremer, P., Mints, G.: Dynamic topological logic. Ann. Pure Appl. Logic 131, 133-158 (2004)

6. Kripke, S.: A completeness theorem in modal logic. J. Symbolic Logic 24, 1-14 (1959)

7. Kripke, S.: Semantical analysis of modal logic I, normal propositional calculi. Z. Math. Logik Grundlagen Math. 9, 67-96 (1963)

8. Kröger, F.: Temporal Logics of Programs. Springer, Berlin Heidelberg New York (1985)

9. McKinsey, J.C.C.: A solution of the decision problem for the Lewis systems S2 and S4, with an application to topology. J. Symbolic Logic 6, 117-134 (1941)

10. McKinsey, J.C.C., Tarski, A.: The algebra of topology. Ann. Math. 45, 141-191 (1944)

11. Mints, G., Zhang, T.: Propositional logic of continuous transformation in Cantor space. Arch. Math. Logic 44, 783-799 (2005)

12. Prior, A.N.: Past, Present and Future. Clarendon Press, Oxford (1967)

13. Segerberg, K.: Von Wright's tense logic. In: Schilpp, P.A., Hahn, L.E. (eds.) The Philosophy of Georg Henrick von Wright, Open Court, La Salle (1989) 603-635 (1974)

14. Segerberg, K.: Discrete linear future time without axioms. Stud. Logica 35, 273-278 (1976)

15. Segerberg, K.: Von Wright's logic of time (in Russian). Logitjeskij Vyvod, Nauka, Moscow (1979)

16. Slavnov, S.: Two counterexamples in the logic of dynamic topological systems. Technical Report TR-2003015, Cornell University, Ithaca (2003)

17. von Wright, G.H.: And next. Acta Philos. Fennica 18, 293-304 (1965)

18. von Wright, G.H.: Always. Theoria 34, 208-221 (1968)

19. Zhang, T., Mints, G.: A proof of topological completeness of $\mathrm{S} 4 \mathrm{C}$ for dynamic Cantor spaces. (manuscript) (2003) 